Review Article

\title{
Positive evidence for P6 acupuncture antiemesis
}

\author{
J.W. Dundee and C. McMillan \\ Northern Ireland Radiotherapy and Oncology Centre, Belvoir Park Hospital, Belfast, and Department of \\ Anaesthetics, The Queen's University of Belfast, Belfast, N. Ireland
}

\section{Introduction}

An antiemetic action is claimed among other uses for acupuncture (ACP), and several antiemetic ACP points are described in textbooks. The most accessible of these is P6, so called because it is on the pericardial meridian. This is also known as Neiguan (Nei-kuan) or G-Jo point no. 10, and the simplest description of locating it is the width of two thumbs above the distal crease on the inner wrist, in line with the middle finger. Having seen acupressure at this point used as a preventative for morning sickness in China one of the authors (JWD) initiated a scientific study of the antiemetic action of stimulation of the P6 point. Concurrent with these studies, which started in 1984, others have carried out similar investigations. We here critically review both our own and other studies on this topic.

While some workers refer to anti-nausea effect, the majority include both nausea and vomiting. Objections to including nausea which is a subjective sensation, are minimized by simply noting its presence and not attempting to quantify it although methods of quantifying it have been described. ${ }^{1}$ Some attempt to quantify the severity of vomiting, based on the number of episodes, while others simply note its occurrence in a given time period. In the cancer chemotherapy field, while some quantify the severity of sickness, the majority of workers look at the reduction in sickness with different forms of therapy.

\section{Methods of stimulation}

Initially manual ACP at the P6 was used, but later studies have involved other methods of stimulation of Neiguan. These can be grouped as follows:

Correspondence: Professor J.W. Dundee, O.B.E., M.D., The Oaks, 24 Old Coach Road, Belfast BT9 SPR, UK. Accepted: 20 November 1990

\section{Invasive}

Acupuncture with manual rotation of needle Acupuncture with electrical stimulation of needle (electro ACP).

\section{Non-invasive}

Transcutaneous electrical stimulation (TCES)

Acupressure - pressure over point.

Electroacupuncture involves a current applied to the needle from a battery-operated DC square wave stimulator, with a variable current output which is adjusted until the patient is aware of the stimulation. The same apparatus is used for TCESo (via surface electrodes) and with both techniques the neutral lead is placed somewhere on the same limb - usually on the back of the hand or on the Hegu point.

Acupressure can be applied manually or by the use of an elasticized band with a large plastic stud which is easily positioned over the Neiguan point. Originally devised by Daniel C. Choy from New York and commonly known as 'Choy Bands' or 'Choy strap' these are now available commercially under the name of Sea Bands.

\section{Clinical uses}

P6 antiemesis has been used in the following fields:

1. Postoperative

2. Morning sickness

3. Cancer chemotherapy

4. Travel sickness.

The findings will be reviewed in the above order. It will become apparent that the only reliable scientific data were obtained in the first group. There have been few studies in sickness of early pregnancy and in travel sickness, while with cytotoxic drug therapy the condition of patients often restricted the use of factors such as randomization and control (no treatment) groups.

Many acupuncture text books and one Chinese paper ${ }^{2}$ do not distinguish between different causes of sickness and these are not referred to here. 


\section{Postoperative sickness}

The most complete study in this field is by the authors and colleagues. ${ }^{3-7}$ This involves over 500 female patients having short (8-12 min) gynaecological operations (cervical dilatation and uterine curettage) under a standard methohexitonenitrous oxide anaesthetic. Premedication was with $10 \mathrm{mg}$ nalbuphine given intramuscularly. Except in one series, P6 was stimulated before administration of the opioid. No drugs were given which are known to affect the incidence of sickness. All four methods of stimulation of P6 were used in random order and a control (no treatment) group was included. In addition a small group had needling of a point near the right elbow which is outside the normal ACP meridians (dummy ACP). Initially all treatments were applied to the right forearm but later this was changed to the dominant side. Patients were visited at the end of the first and sixth postoperative hours, by a person who was unaware of the treatment given, when the occurrence of nausea alone (N) or vomiting (including retching) with or without nausea (V) was noted. All were told that the study was designed to reduce side effects and improve the efficacy of the premedication; nausea and vomiting were not mentioned at this stage, but a full description of the study objectives was given at the time of the last postoperative visit. There was a minimum of 31 patients in each group.

Figure 1 summarizes the findings in all the Belfast studies. The incidence of sickness was reduced to a significant degree $(P<0.001)$ by both

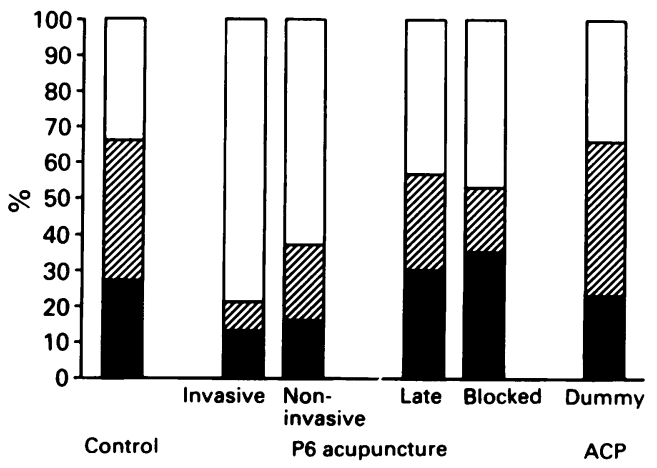

Figure 1 Percentage incidence of vomiting (with or without nausea) or nausea alone $\mathbb{Z M}$ in the first 6 hours after operation in patients having minor operations under a fixed premedicant and anaesthetic regime. Patients either received no adjuvant therapy (controls) or invasive or non-invasive stimulation of P6 acupuncture point before administration of the premedicant. A point near the right elbow was also stimulated (Dummy ACP). In one group (Late) the ACP was given in the operating theatre while in another (Blocked) the ACP site was infiltrated with lignocaine. invasive and non-invasive stimulation of $\mathrm{P} 6$, but not by stimulation of the 'dummy point'. The non-invasive approach was not as effective $(P<$ 0.01 ) as the invasive, the difference being in the duration of action, both being equieffective in the first postoperative hour, but the benefit of both TCES and acupressure was less effective in the 1-6 hour period. With electrical stimulation of the ACP needle and TCES best results were obtained with $10-15 \mathrm{~Hz}$ applied for $5 \mathrm{~min}$.

In a subsequent investigation using the same study model, it was found that infiltration of the acupuncture site with lignocaine abolished the antiemetic action of Neiguan stimulation compared with subjects in whom the injection was with saline. $^{8}$

Contemporaneously with the first Belfast study Fry9 reported an antiemetic action from acupressure applied in the immediate post-anaesthetic period and commented on its brevity of action. At the same time, in a small but well designed study in patients having eye surgery, Masuda and colleagues ${ }^{10}$ noted a reduction in postoperative sickness in those having acupuncture carried out at the time of induction of neurolept anaesthesia as compared with untreated controls.

Two studies in which acupuncture was given during an opioid-containing anaesthetic failed to demonstrate any antiemetic action. ${ }^{11,12}$ These suggest that there may be a strong psychological element in acupuncture antiemesis. However, Dundee and Ghaly ${ }^{13}$ have shown that the timing of ACP in relation to the administration of the emetic stimulus is important. Using the same nalbuphine-operation model they failed to demonstrate antiemetic activity when ACP was carried out immediately before induction or during anaesthesia as compared with a good effect when given before the opioid (Figure 1).

In a well controlled (female patients, standard operation) perspective study which involved 4 anaesthetic techniques $\mathrm{Ho}$ and colleagues ${ }^{14}$ demonstrated a significant antiemetic action from P6 acupuncture in patients whose anaesthesia included an opioid. The reduction in incidence was similar to that produced by $5 \mathrm{mg}$ prochlorperazine intravenously. These workers did not include nausea in their study, but they did find ACP to be better than TCES.

The most recent study by Barsoum and colleagues $^{15}$ investigated the benefit of acupressure (Sea Bands) at P6 point in relieving sickness following 162 major operations. Patients received a variety of premedicants and anaesthetics but postoperative analgesia was standardized (papaveretum). Adjuvant therapy, (acupressure, 'dummy' Sea Bands and prochlorperazine) was given in random order and one group received no treatment. Nausea scores (as assessed on a linear analogue scale) were 
significantly lower in the first two postoperative days in those patients treated by acupressure when compared with the other groups. Vomiting was recorded in fewer patients amongst the acupressure groups than in the control or drug group, but the difference was not statistically significant.

\section{Morning sickness}

When acupressure at P6 was practised in an obstetric outpatient clinic in Beijing the mothers were told that 'it always works'. Supporting evidence for this could not be obtained, but the remedy has been used for 4000 years and is quoted in most acupuncture text books. Two recent inadequately documented Chinese studies claim benefit from needling a number of points including Neiguan in patients with morning sickness claiming 'fairly satisfactory results' without giving any details. ${ }^{16,17}$ A Russian paper is likewise lacking in data $^{18}$ as is one from Czechoslovakia. ${ }^{19}$

The beneficial effect of self-administered acupressure has been studied in 350 consecutive women attending a Belfast antenatal clinic. ${ }^{20} \mathrm{~A}$ self-reporting system of study was adopted, patients being given either no treatment, told to press P6 for 5 minutes every 2 to 3 hours, or to press a dummy point at the right elbow. Although the incidence of returned records $(70 \%)$ was less than hoped for, the findings in Table I show benefit from P6 acupressure $(P<0.0005)$, with lesser benefit $(P<0.01)$ from pressing the 'dummy' point. Since patients had to have the reason for pressing their elbow explained to them, the latter would appear to be a psychological effect. This study was far from ideal, and has been criticized for low incidence of returned records - a factor outside the control of the workers. However, it is not without merit and the authors have successfully used self-administered 2-hourly transcutaneous electrical stimulation of P6 in over 20 patients with marked success.

A more scientifically controlled clinical trial, limited only by the small numbers involved, has been reported by an American nurse-midwife. ${ }^{21}$ Commercially available wrist bands with a stud pressing on P6 were worn for 5 days followed by a similar band without a stud, the order being reversed in half the patients. Using a visual analogue scale a significant reduction in nausea was demonstrated $(P<0.05)$ from acupressure with relief of symptoms in 12 of 16 subjects.

\section{Oncology}

The emetic effect of cisplatin and other potent cytotoxic drugs is well known, and, until the introduction of the $5 \mathrm{HT}_{3}$ antagonists, treatment of this was not very effective. ${ }^{22-26}$
Table I Percentage incidence of varying degrees of morning sickness in antenatal patients applying acupressure at $\mathrm{P} 6$ point or at the right elbow and in a group having no treatment

\begin{tabular}{lcccc}
\hline Acupressure & $n$ & Severe & Moderate & Nil \\
\hline Nil & 119 & 56 & 21 & 23 \\
P6 & 110 & 19 & 21 & 60 \\
Right elbow & 112 & 37 & 37 & 26 \\
\hline
\end{tabular}

Adapted from Dundee, Sourial, Ghaly \& Bell, $1988 .{ }^{20}$

In cancer treatment centres, the reported incidence of troublesome vomiting is in the region of $56 \%{ }^{27}$ to $76 \% .^{28}$ In the latter survey $96 \%$ of patients who were sick with one course of treatment experienced the same on the second occasion. This was the setting for the reported Belfast studies of the antiemetic action of $\mathrm{P} 6$ acupuncture in patients receiving cancer chemotherapy. ${ }^{28,29}$ Those who had troublesome sickness with a previous course of therapy, despite the use of standard antiemetics, had ACP before starting the next course with continuation of their antiemetics. At that time these were metoclopramide, cyclizine and some phenothiazines (prochlorperazine, thiethyperazine and chlorpromazine) with lorazepam and steroids as adjuvants.

Before discussing the findings, one must mention the limitations of the studies. Firstly, patients knew they were being referred for a 'new treatment for sickness' and even when ACP was not mentioned, patients talked among themselves and the subject had good media coverage. Secondly, ethical considerations limited the use of a 'dummy point' technique, to very few patients. Assessment of benefit was based on the opinion of the patient and attendants, all of whom knew the treatment that had been given. The four point scale for benefit attributed to $\mathrm{ACP}$ did not attempt to quantify the degree of nausea or vomiting. ${ }^{28}$ Both hospitalized and outpatients were studied; daily visits to the former gave more reliable data than that obtained from outpatients on their next visit to the clinic, which was normally 3-4 weeks after previous treatment, although these were often contacted by phone. The input by the nursing staff on the severity and frequency of sickness also made the inpatient data more reliable.

The initial studies were with manual or electrical $\mathrm{ACP}^{28}$ and these were followed by non-invasive approaches to stimulation of $\mathbf{P} 6,{ }^{29}$ including selfadministration of TCES ${ }^{30}$ The findings in all series are summarized in Figure 2 which is based on about 1,000 treatments in more than 200 patients. Best results were obtained with invasive ACP which benefited more than $90 \%$ of patients although the effect often only lasted for about 8 hours. The 
beneficial effects were marginally, but not significantly, less in patients having highly emetic compounds as compared with other forms of chemotherapy. In a small blind crossover study, only one out of 10 patients benefited from 'dummy ACP' as compared with 9 from P6 stimulation. ${ }^{28}$

Indirect electrical stimulation (TCES) had many practical advantages over an invasive approach with $85 \%$ patients benefiting from it. By the use of large diffuse low impedance (ECG) electrodes, it could easily be administered by patients themselves. ${ }^{30}$

While 2-hourly acupressure prolonged the beneficial effect of both ACP and TCES, better results were obtained when TCES was used every 2 hours. In this series acupressure alone was not very effective, but, in a smaller study, Stannard found that the wearing of Sea Bands reduced nausea. ${ }^{31}$

Italian workers ${ }^{32}$ have confirmed the Belfast findings with ACP. They found both increased complete protection from nausea and a decrease in the intensity and duration of vomiting.

It must be stressed that in all the chemotherapy studies, stimulation of P6 has proved a useful addition to standard antiemetics, rather than a treatment on its own.

\section{Motion sickness}

Only acupressure has been used in this field employing either the Choy strap or Sea Bands. The idea arose from the personal experience of Dr Choy in the Newport-Bermuda boat race in 1980. Pressure on the Nei-kuan point, although effective in relieving his nausea, left him with only one hand to sail his $\mathbf{4 0}$ foot sloop. ${ }^{33}$ Most of the supportive evidence is anecdotal including a strong recommendation in the press. ${ }^{34}$

There have been 2 scientific investigations into the efficacy of these acupressure bands. ${ }^{35,36}$ Both

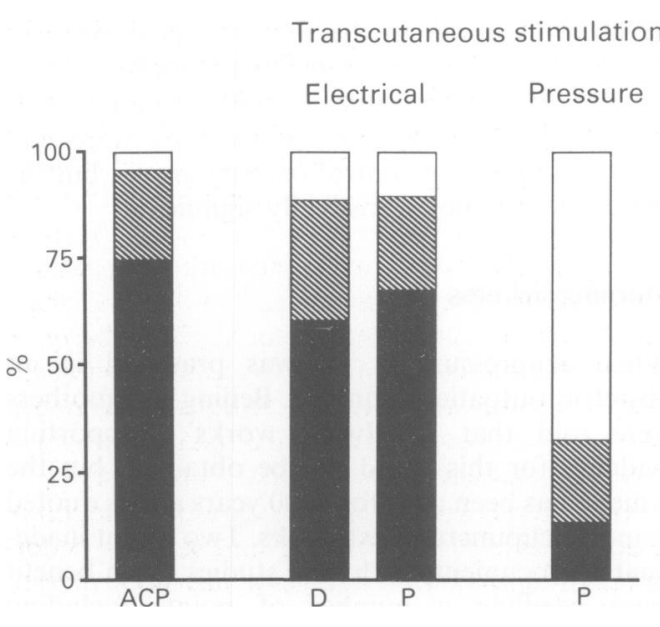

Figure 2 Percentage incidence of good $\square$ moderate $\square$ and poor $\square$ benefit obtained by various methods of stimulation of P6. ACP = acupuncture; $D=$ doctor administered; $\mathbf{P}=$ patient administered.

employed a motion challenge test and neither found Sea Bands to be effective.

Table II is an attempt to summarize all the above data.

\section{Discussion}

Acupuncture literature refers to other sites from which an antiemetic action is claimed. These include Hegu (at the base of the thumb), and Tsu-San-Li (S36) and Feng-Lung (S40), both of which are in the lower limbs. Ease of access and simplicity of technique has presumably led to all studies being limited to stimulation of the P6 point.

Under certain circumstances, there is undoub-

Table II Summary of evidence of antiemetic action of stimulation of Neiguan point

\begin{tabular}{ll}
\hline Postoperative sickness & $\begin{array}{l}\text { : Good experimental and clinical evidence of efficacy. ACP and } \\
\text { TCES equieffective, but ACP benefit lasts longer. } \\
\text { Must be given before emetic stimulus. }\end{array}$ \\
Morning sickness & $\begin{array}{l}\text { : Acupressure only studied. Some benefits claimed. } \\
\text { Some psychological element in benefit. }\end{array}$ \\
Cancer chemotherapy & : P6 stimulation given in conjunction with standard antiemetics \\
& which alone had proved ineffective. Good clinical evidence for \\
efficacy. & ACP better than TCES. Acupressure alone is not very effective, \\
& but prolongs action of ACP and TCES. \\
Travel sickness & Acupressure only studied. \\
& No scientific, but much anecdotal evidence of efficacy.
\end{tabular}


tedly a psychological element in the antiemetic action of acupuncture. This probably played a part in the reported studies of morning sickness where stimulation of a 'dummy' point had some antiemetic action. It may also have been a contributory factor in the cancer chemotherapy studies where patients knew what to expect. However, in both postoperative and cancer chemotherapy studies stimulation of a 'dummy ACP' point or 'sham ACP' had no antiemetic action. The ability to 'block' the antiemetic action, by infiltration of the Neiguan point with local anaesthesia, ${ }^{8}$ also points to a non-psychological action. Similar observation has been made in respect to ACP analgesia. ${ }^{37,38}$

Very little is known about the mode of action of acupuncture. ${ }^{39,40}$ The role of endogenous endorphins in acupuncture analgesia has been investigated with contradictory results. Some report antagonism of analgesia by naloxone $e^{41,42}$ while others found no antagonism. ${ }^{43,44}$ The consensus of opinion appears to favour the view that endogenous endorphins are released by the acupuncture, but this is unlikely to apply to its antiemetic action.

In general it appears that a lower frequency of stimulation is required for an antiemetic as compared with an analgesic action, ${ }^{7,14,45}$ but even this view is not universally accepted. ${ }^{46}$ In a study of

\section{References}

1. Del Favero, A., Roila, F. \& Basurto, C. et al. Assessment of nausea. Eur J Clin Pharmacol 1990, 38: 115-120.

2. Deng, D.H., Tan, O.L. \& Han, J.S. Observations on combating nausea by finger pressure on the Hegu point. $J$ Tradit Chin Med 1986, 6: 111-112.

3. Bill, K.M. \& Dundee, J.W. Acupuncture for postoperative nausea and vomiting. Br J Clin Pharmacol 1989, 26: 225P.

4. Dundee, J.W., Chestnutt, W.N., Ghaly, R.G. \& Lynas, A.G.A. Traditional Chinese acupuncture: a potentially useful antiemetic? $\mathrm{Br}$ Med J 1986, 293: 583-584.

5. Dundee, J.W., Ghaly, R.G., Bill, K.M., Chestnutt, W.N., Fitzpatrick, K.T.J. \& Lynas, A.G.A. Effect of stimulation of the P6 antiemetic point on postoperative nausea and vomiting. $B r J$ Anaesth 1989, 63: 612-618.

6. Dundee, J.W., Ghaly, R.G. \& McKinney, M.S. P6 acupuncture antiemesis: comparison of invasive and non-invasive techniques. Anesthesiology 1989, 71: A130.

7. Ghaly, R.G., Fitzpatrick, K.T.J. \& Dundee, J.W. Antiemetic studies with traditional Chinese acupuncture: a comparison of manual needling with electrical stimulation and commonly used antiemetics. Anesthesia 1987, 42: 1108-1110.

8. Ghaly, R.G. \& Dundee, J.W. Are neural pathways involved in acupuncture antiemesis? Irish J Med Sci 1989, 159: 30.

9. Fry, E.N.S. Acupressure and postoperative vomiting. Anesthesia 1986, 41: 661-662.

10. Masuda, A., Miyazaki, H., Yamazki, M., Pintov, S. \& Ito, Y. Acupuncture in the anesthetic management of eye surgery. Acupuncture Electro Res 1986, 11: 259-267.

11. Weightman, W.M., Zacharias, M.\& Herbison, P. Traditional Chinese acupuncture as an antiemetic. $\mathrm{Br} \mathrm{Med} J$ 1987, 295: $1379-1380$. acupuncture as a prophylaxis for migraine headaches, Lenhard and Waite ${ }^{47}$ found a decrease in sickness without any analgesia. This suggests a different mechanism for the two actions of acupuncture. Harris has suggested that cytotoxic-therapy-induced vomiting is mediated via enkephalic pathways ${ }^{48}$ which would preclude an endorphinmediated action for ACP antiemesis.

It is many years since Borison and Wang ${ }^{49}$ demonstrated the existence of a vomiting centre which receives stimuli from various sites including the chemoreceptor trigger zone (CTZ), the gastrointestinal tract, and cerebral cortex. The CTZ, located on the floor of the 4th ventricle within the area postrema senses chemical stimuli which can provoke emesis when present in the blood in sufficient concentration. Based on the response to therapy a number of possible neurotransmitters have been postulated: receptors for dopamine serotonin, cholinergic and possibly adrenergic and histaminergic drugs may be involved in the transmission of neurochemical signals. ${ }^{50,51}$ This may explain the efficacy of a five drug regime in preventing sickness induced by cisplatin combination therapy. ${ }^{52.53}$ The synergistic effect of P6 stimulation with standard antiemetics suggests that it may be acting on another, as yet unknown neurotransmitter.

12. Dundee, J.W., Milligan, K.R. \& McKay, A.C. The influence of intraoperative acupuncture and droperidol on postoperative emesis. Br J Anaesth 1988, 61: 116-117.

13. Dundee, J.W. \& Ghaly, R.G. Does the timing of P6 acupuncture influence its efficacy as a postoperative antiemetic? Br J Anaesth 1989, 63: 630P.

14. Ho, R.T., Jawan, B., Fung, S.T., Cheung, H.K. \& Lee, J.H Electro-acupuncture and postoperative emesis. Anesthesia 1990, 45: 327-329.

15. Barsoum, G., Perry, E.P. \& Fraser, I.A. Postoperative nause is relieved by acupressure. $J R$ Soc Med 1990, 83: 86-89.

16. Changxin, $Z$. Acupuncture treatment of morning sickness. $J$ Tradit Chin Med 1988, 8: 228-229.

17. Rongjun, Z. 39 cases of morning sickness treated with acupuncture. J Tradit Chin Med 1987, 7: 25-26.

18. Osadchaia, O.V. \& Shabadash, V.V. Changes of central nervous system function in patients with hyperemesis gravidarum treated by acupuncture. Akush-Ginerol (Mosk) 1989, 5: $55-56$.

19. Jindrak, M. Treatment of vomitus matutinus and emesis gravidarum by acupuncture in clinical practice. Cesk Gyneko 1980, 45: 303-304.

20. Dundee, J.W., Sourial, F.B.R., Ghaly, R.G. \& Bell, P.F. P6 acupressure reduces morning sickness. $J R$ Soc Med 1988, 81: 456-457.

21. Hyde, E. Acupressure therapy for morning sickness. A controlled clinical trial. $J$ Nurse-Midwifery 1989, 34: $171-178$ 
22. Gralla, R.J., Itri, L.M. \& Pisko, S.E. et al. Antiemetic efficacy of high-dose metoclopramide: randomised trials with placebo and prochlorperazine in patients with chemotherapy-induced nausea and vomiting. $N$ Engl $J$ Med 1981, 305: 905-909.

23. Fetting, J.H., Grochow, L.B., Folstein, M.F., Ettinger, D.S. $\&$ Colvin, $\mathbf{M}$. The course of nausea and vomiting after high dose cyclophosphamide. Cancer Treat Rep 1982, 66: 14871493.

24. Grunberg, S.M. Making chemotherapy easier. N Engl J Med 1990, 322: 846-848.

25. Kirkham, S.R. Palliative care of the patient with advanced cancer. Cancer Treat Rev 1989, 16: 119-128.

26. Priestman, T.J. Cancer Chemotherapy: an Introduction. Springer-Verlag, Berlin, Heidelberg, 1989.

27. Hoskins, P.J. \& Hanks, G.W. The management of symptoms in advanced cancer: experience in a hospital-based continuing care unit. J R Soc Med 1988, 81: 941-944.

28. Dundee, J.W., Ghaly, R.G., Fitzpatrick, K.T.J., Abram, W.P. \& Lynch, G.A. Acupuncture prophylaxis of cancer chemotherapy-induced sickness. $J R$ Soc Med 1989, 82 $268-271$

29. Dundee, J.W. \& Yang, J. Prolongation of the antiemetic action of $\mathbf{P 6}$ acupuncture by acupressure in patients having cancer chemotherapy. $J R$ Soc Med 1990, 83: 360-361.

30. McMillan, C. \& Dundee, J.W. Problems of self-administration of P6 (Neiguan) antiemesis. Br J Clin Pharmacol 1991, 31: 236P.

31. Stannard, D. Pressure prevents nausea. Nursing Times 1989, 85: 33-34.

32. Aglietti, L., Roila, F. \& Tonato, M. et al. A pilot study of metoclopramide, dexamethasone, diphenhydramine and acupuncture in women treated with cisplatin. Cancer Chemother Pharmacol 1990, 26: 239-240.

33. Choy, D.S.J. Personal communication. 1990.

34. Fishman, J.A. Seasickness cure: It's all in the wrists. New York Times 1981; March 15.

35. Lentz, J.M. Two experiments on laboratory induced motion sickness I. acupressure: II. repeated exposure. Pensacola FL: US Naval Aerospace Medical Research Laboratory Report 1982.

36. Bruce, D.G., Golding, J.F., Hockenhull, N. \& Pethybridge, R.J. Acupressure and motion sickness. Aviat Space Environ Med 1990, 61: 361-365.

37. Chiang, C.Y., Liu, J.Y., Chu, T.H., Pai, Y.H. \& Chang, S.C. Studies on spinal ascending pathway for effect of acupuncture analgesia in rabbits. Scientia Sinica 1975, XVIII: $651-658$.
38. Lu, G., Liang, R., Xie, J., Wang, Y. \& He, G. Role of peripheral afferent nerve fiber in acupuncture analgesia elicited by needling point Zusanli. Scientia Sinica 1979, XXII: 680-692.

39. Vincent, C.A. \& Richardson, P.H. The evaluation of therapeutic acupuncture: concepts and methods. Pain 1986, 24: $1-13$.

40. Richardson, P.H. \& Vincent, C.A. Acupuncture for the treatment of pain: a review of evaluated research. Pain 1986 , 24: $15-40$.

41. Hosobuchi, Y., Adams, J.E. \& Linchitz, R. Pain relief by electrical stimulation of the central grey matter in humans and its reversal by naloxone. Science 1977, 197: 183-186.

42. Mayer, D.J., Price, D.D. \& Rafii, A. Antagonism of acupuncture analgesia in man by the narcotic antagonist naloxone. Brain Res 1977, 121: 368-372.

43. Lindblom, V. \& Tegner, R. Are the endorphins active in clinical pain states? Narcotic antagonism in chronic pain patients. Pain 1979, 7: 65-68.

44. Chapman, C.R., Colpitts, Y.M., Benedetti, C., Kitaeff, R. \& Gehring, J.D. Evoked potential assessment of acupunctural analgesia: attempted reversal with naloxone. Pain 1980, 9: $183-197$.

45. Johnson, M.I., Ashton, C.H., Bousfield, D.R. \& Thompson, J.W. Analgesic effects of different frequencies of transcutaneous electrical nerve stimulation on cold-induced pain in normal subjects. Pain 1989, 39: 231-236.

46. Baldry, P.E. Acupuncture, Trigger Points and Musculoskeletal Pain. Churchill Livingstone, Edinburgh, London, 1989.

47. Lenhard, L. \& Waite, P.M.E. Acupuncture in the prophylactic treatment of migraine headaches: pilot study. $N Z \mathrm{Med}$ $J$ 1983, 96: 663-666.

48. Harris, A.L. Cytotoxic-therapy induced vomiting is mediated via enkephalic pathways. Lancet 1982, i: 714-716.

49. Borison, H.L. \& Wang, S.G. Physiology and pharmacology of vomiting. Pharmacol Reviews 1953, 5: 193-230.

50. Peroutka, S.J. \& Synder, S.H. Antiemetic neurotransmitter receptor binding predicts action. Lancet 1982 , i: 658-659.

51. Borison, H.L. \& McCarthy, L.E. Neuropharmacology of chemotherapy-induced emesis. Drugs 1983, 25: 8-17.

52. Editorial. The chemoreceptor trigger zone revisited. Lancet 1987, i: 144.

53. Kessler, J.F., Alberts, D.S. \& Plezia, P.M. et al. An effective five-drug antiemetic combination for prevention of chemotherapy-related nausea and vomiting. Cancer Chemother Pharmacol 1986, 18: 282-286. 